\title{
Changing a protocol form to list animal number by strain
}

At Great Eastern University, investigators using animals were required to list on the IACUC protocol form the number of animals, by species, being requested. Although this requirement was well-established at Great Eastern and many other institutions, it was questioned by the school's IACUC administrator, who believed it would be more appropriate to list on the IACUC protocol form the number of animals requested by strain and species, rather than by species alone. Her rationale was simple: many research protocols at Great Eastern required one or more specific strains of mice, and she felt that failure to list the number of animals needed for each strain could lead to the acquisition of more animals than needed, animals that could be potentially unusable. For example, she said, "If 100 mice were required for a study, and 50 of them had to be $\mathrm{C} 57 \mathrm{Bl} / 6$, how would the IACUC know whether the investigator used only the approved 50 rather than $100 \mathrm{C} 57 \mathrm{Bl} / 6$ mice, if only the species was indicated on the protocol? And, if $100 \mathrm{C} 57 \mathrm{BL} / 6$ were obtained, what were the extra 50 used for?"
Of course, not everybody agreed with that reasoning, particularly the researchers on the committee. They presented four points of view against having to list specific strains on the protocol form. First, researchers would never spend their precious research grant dollars purchasing animals they did not need. Second, the Animal Welfare Act regulations ${ }^{1}$ and the Public Health Service Policy on Humane Care and Use of Laboratory Animals ${ }^{2}$ indicate a need to justify the approximate number of animals required by species, not by any additional descriptions. Third, research needs change and researchers would not want to waste time submitting protocol amendments to change the number of animals of a particular strain requested. And fourth, noncompliance is noncompliance, no matter which species, strain or stock is being used. Nevertheless, some other members of the IACUC took up the administrator's cause, reminding the investigators that when multiple strains were to be used in a protocol, the information about the strains was already included in the descriptive parts of the protocol form in order for the reviewers to understand the component parts of the planned study. They also noted that when animals were eventually ordered, the needed strain had to be indicated. Therefore, they wanted to know why it would be a problem to list strain information on the protocol form where the species and total number of animals requested was shown. That way, they claimed, there would be less chance for ordering errors and less chance for noncompliance.

What is your opinion? Do you think Great Eastern University should change the protocol form along the lines suggested by the IACUC administrator or leave it as it is, as favored by the researchers?

1. Animal Welfare Act and Regulations. 9 CFR Chapter 1, Subchapter A.

2. Public Health Service. Policy on Humane Care and Use of Laboratory Animals (US Department of Health and Human Services (US Department of Health and Human Services, Washington, DC, 1986; amended 2002).

\section{RESPONSE}

\section{Right info, wrong place}

\section{Holly McEntee, MA, CPIA}

The purpose of the protocol form is to collect the information required by law to enable the IACUC to make an informed decision about the proposed use of animals, balancing potential benefits of the proposed use against the potential and real pain and distress experienced by the animals. Two pieces of information the IACUC needs to make this decision are the identification of the species and the approximate number of animals to be used (section 2.31 (e)(1) of the Animal Welfare Act regulations $)^{1}$. Because the Public Health Service Policy on Humane Care and Use of Laboratory Animals ${ }^{2}$ requires compliance with the Animal Welfare Act regulations, investigators must identify for the IACUC all vertebrate species to be used in the protocol-not only those species covered by the Animal Welfare Act-and the approximate number of each species to be used. For the purposes of IACUC oversight and evaluation of proposed and ongoing animal use, neither regulatory document requires the provision of information beyond species identification.
I would not advocate for listing specific strains and numbers of animals requested by strain on the IACUC animal protocol form. In my opinion, too often institutions try to use the IACUC animal care and use protocol application to fulfill institutional responsibilities beyond that of informing the IACUC's decisions. A well-coordinated institutional animal program should have systems and processes that proactively prevent the ordering of more animals than is approved by the IACUC or that identify noncompliance if more animals were ordered than were approved by the IACUC. The approved IACUC protocol can be a source document to inform those systems 